EPiC Series in Engineering
Volume 3, 2018, Pages 1639-1647
HIC 2018. 13th International
Conference on Hydroinformatics

\title{
Comparison between diffusive and advective approach in quality analysis of a real distribution network
}

\author{
Stefania Piazza ${ }^{1}$, E. J. Mirjam Blokker ${ }^{2}$, Gabriele Freni ${ }^{1}$, Valeria Puleo ${ }^{1}$ \\ and Mariacrocetta Sambito ${ }^{1}$ \\ ${ }^{1}$ School of Engineering and Architecture, University of Enna "Kore", Cittadella Universitaria, 94100 \\ Enna, Italy \\ ${ }^{2}$ KWR Watercycle Research Institute, P.O. Box 1072, 3430 BB Nieuwegein, The Netherlands \\ gabriele.freni@unikore.it
}

\begin{abstract}
In recent years, the evaluation of water quality in distribution systems has generated enormous interest in the scientific community due to the increasing concentration of population in urban areas and frequent issues connected with supply water quality. Following the wave of bioterrorism subsequent the events of September 11th 2001, a need can be foreseen to seek adequate preventive measures to deal with contamination in water distribution systems that may be related to the accidental contamination and deliberate injection of toxic agents of any origin in the distribution networks. Therefore, it is very important to create a sensor system that detects contamination events in real time, while maintaining the reliability and efficiency of the measurements, limiting the cost of the instrumentation. A reliable monitoring system, for this kind of problems, cannot be deployed without realistic modelling support. The current state-of-the-art in water distribution systems analysis usually adopt a simplified approach to water quality modelling, neglecting dispersion and diffusion and considering simplified reaction kinetics. Even if such simplifications are commonly acceptable in fully turbulent flows, they may take to relevant errors in transition flows with low velocity thus taking to unreliable interpretation of the contamination in complex networks. The present paper aims to compare different modelling approaches to the evaluation of contaminant dispersion in two distribution networks: one laboratory network in which contamination experiments were carried out in a controlled environment (Enna, Italy) and a full-scale real distribution network (Zandvoort, Netherlands).
\end{abstract}




\section{Introduction}

Water distribution systems connect consumers to water resources, using hydraulic components such as pipes, valves and tanks that are usually the most vulnerable elements, as they may represent a pathway for contamination intrusion.

Adequate water quality in distribution networks is a fundamental requirement that must be guaranteed to safeguard public health. The aim of maintaining water quality standards is important for the entire integrated water system (Freni \& Sambito, 2017). Water quality monitoring is an indispensable pre-requisite and it can be reached using a variety of methods. Several studies have been carried out in the literature on the optimal positioning of sensors, using different methods of optimization, such as standard genetic algorithm (GA) (Ozdemir \& Ucaner, 2005) (Tolson, et al., 2004), non-dominated sorting genetic algorithm NSGA-II (Preis \& Ostfeld, 2008), linear programming algorithm (LP) (Boccelli, et al., 1998), Multi-Layer Perceptron (MLP) and General Regression Neural Network (GRNN) (Gibbs, et al., 2006).

All the above-mentioned studies rely on models to present a robust estimation of contaminant distribution and concentrations. The use of simplified models may take to unreliable estimation of contaminant propagation thus taking to the deployment of inefficient monitoring networks (Piazza, et al., 2017).

The great majority of literature researches are based on hydraulic simulation tools, such as EPANET, that adopt a simplified approach to water quality based on advective transport and some simplified reaction kinetics.

Even if such simplifications are adequate for many practical applications, dispersive / diffusive transport processes become relevant when flow velocity are low and Reynolds numbers are under 50000 , like frequently in urban water distribution networks during night (Axworthy \& Karney, 1996). Moreover, optimization studies were often based on numerical and modelling analysis without any comparison with experimental data.

Many authors have dealt with the problem of advection-dispersion transport. Dong at al. (2008) (Dong \& Selvadurai, 2008) have developed a model capable of modelling the advection dominated transport process. Pérez Guerrero et al. (2013) (Pérez Guerrero, et al., 2013) have extended the Duhamel theorem, originally established for diffusion type problems, to the case of advective-dispersive transport subject to transient (time-dependent) boundary conditions. As the analytical solution of the advection-dispersion solute transport equation remains useful for a large number of applications in science and engineering, several studies have concentrated in this direction. Berger et al. (2017) (Berger, et al., 2017) proposed to solve the advection-diffusion differential equation trough two numerical schemes, Scharfetter-Gummel and Crank- Nicolson approach, whose efficiencies were investigated for both linear and nonlinear cases. Li et al. (2011) (Li \& Cleall, 2011) have studied analytical solutions for advection and dispersion of a conservative solute in a one-dimensional double-layered finite porous media are presented. Williams et al. (2008) (Williams \& Tomasko, 2008) have presented an analytical solution of the one-dimensional contaminant transport undergoing advection, dispersion, sorption, and first-order decay, subject to a first-order decaying contaminant concentration at the source and a Type I, Dirichlet, boundary at infinity.

As the general solution of the advective-dispersive problem was largely discussed in literature, applications on pipe networks are piecemeal due to the complexity of replicating analytical solutions for each pipe in the network with concatenating boundary conditions.

After a short discussion of the proposed modelling approach, the present study focuses of weak points of the state-of-the-art distribution networks water quality models: the use a simplified numerical transport model, not able to consider dispersion, and the absence of experimental validation in low Reynolds regimes.

According to this aim, contamination experiments were run, using a conservative tracer, in the laboratory water distribution network of University of Enna "Kore" (Italy) and a field validation on real 
water distribution network in Zandvoort (Netherlands) ) was re-evalueted. The results of a state-of-theart advective model and a diffusive - dispersive model were compared.

\section{Materials and Methods}

The modelling analysis was carried out using the state-of-the-art advective EPANET model (standard advective model) and using an upgraded version of the model (diffusive-dispersive-advective model) including diffusion and dispersion equations proposed by Romero-Gomez et al. (2011) (RomeoGomez \& Choi, 2011).

The authors implement two different equations to take into account the effect of flow direction on dispersion. This approach was used in the study, as it is able to highlight the difference between mass flows, backward and forward from a specific position, resulting from the different dispersion velocities leading to the transport of the solute in the two directions (eq. 1):

$$
\frac{\partial C}{\partial t}=\frac{1}{\Delta x}\left(\phi_{b}-\phi_{f}\right)-u_{m} \frac{\partial C}{\partial x}
$$

in which

$$
\begin{aligned}
& \phi_{b}=-\left.E_{b} \frac{\partial C}{\partial x}\right|_{b} \quad \text { and } \quad \phi_{f}=-\left.E_{f} \frac{\partial C}{\partial x}\right|_{f} \\
& E_{b}=E_{b}(0) \exp (-16 T)+\beta_{b}(T) E^{*} \quad \text { and } \quad E_{f}=E_{f}(0) \exp (-16 T)+\beta_{f}(T) E^{*}
\end{aligned}
$$

where $E_{b}$ and $E_{f}$ (eq. 3) are the dispersion parameters backward and forward with the respect to the flow direction, $u_{m}$ is the flow average velocity and $\beta_{b}(T)$ and $\beta_{f}(T)=1-\exp (-16 T)$.

The dimensionless travel time (T) is determined as follows (eq. 4). This parameter indicates the extent to which the dispersion coefficient has elapsed towards achieving stability conditions.

$$
T=\frac{4 D_{A B} \bar{t}}{d^{2}}=4 \frac{x^{*}}{S_{C} \cdot R}
$$

in which

$$
x^{*}=\frac{L}{d} \text { dimensionless pipe length to define the location of solute migration, } \mathrm{L} \text {, with respect }
$$
to the pipe diameter, $\mathrm{d}$;

$R=\frac{u_{m} \cdot d}{v}$ Reynolds number to account for the mean flow velocity's (um) geometric dimensions (d) and conveying fluid properties (kinematic viscosity, $v$ );

$S_{C}=\frac{v}{D_{A B}}$ Schmidt number to accommodate the solute properties (solute diffusion coefficient, DAB);

$\bar{t}=\frac{L}{u_{m}}$ time defined as the ratio between location of solute migration, L, and flow velocity's (um). 
The models were applied to the experimental network of Enna University - UKE (see (De Marchis, et al., 2016) and (Piazza, et al., 2017) for details, operating the network as a single long loop (Figure 1), and to a real operational water network in the Netherlands, described in (Blokker, et al., 2010).

The laboratory network is a closed water supply distribution network, made up of 3 loops (only one was open in the experimental analysis), 10 nodes and 11 pipes of DN $63 \mathrm{~mm}$, thickness $5.8 \mathrm{~mm}$ and about $45 \mathrm{~m}$ long, arranged in almost horizontal concentric circles with curves having radius $2.0 \mathrm{~m}$; the network is supplied by four tanks, which can store up to $8 \mathrm{~m} 3$ of water. The supply tanks are connected to a group of four pumps and then to an air vessel in order to stabilize pressure. The pumping system behaves like a constant load tank, keeping the pressure constant and equal to a pre-set value, between 1 and 6 bar, with a tolerance of 0.05 bar, varying the speed of the pumps.

System flows in pipes are monitored by 4 electromagnetic flow meters installed in some sections. Pressure cells and multi-jets water meters are present in each node. Additionally, WiFi real time remote controlled conductivity probes were positioned at each node and connected with all the monitoring appliances to a central computer also able to regulate flows supplied to the users by means of remotely controlled valves. Further details about the laboratory network can be found in (De Marchis, et al., 2016).

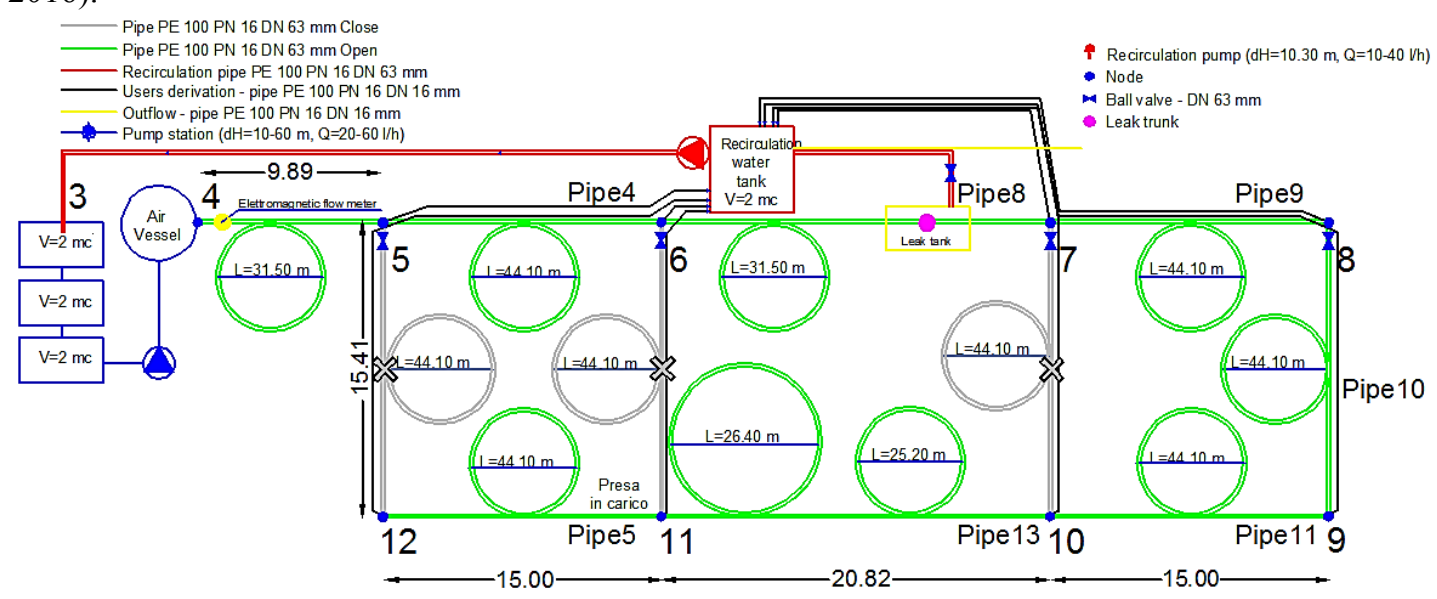

Figure 1: Layout of the water distribution network considering an online operation

The real network is situated in the town of Zandvoort, in the northwest of the Netherlands, along the sea (near Haarlem). The network was built in the 1950-1960s and consists of $5.7 \mathrm{~km}$ of Ø100 mm lined cast iron pipes and $3.5 \mathrm{~km}$ of $\varnothing 100 \mathrm{~mm}$ PVC pipes and supplies 1000 homes, 2 hotels and 30 beach clubs. A tracer study with $\mathrm{NaCl}$ was performed between 2 September and 20 October 2008.

Contamination was simulated by dosing sodium chloride $(\mathrm{NaCl})$ within a booster location nearby the network inlet, raising the electrical conductivity (EC) from $\mathrm{EC} \approx 57 \mathrm{mS} / \mathrm{m}$ without dosage to $\mathrm{EC} \approx$ $68 \mathrm{mS} / \mathrm{m}$. Short intermittent contamination events (3 hours) were performed with an inter-event time of 20 hours. The contamination was carried out for 7 weeks. Electrical Conductivity (EC) values were measured at 4 locations in the system and water age was determined from EC (Blokker, et al., 2010).

In the upgraded version of EPANET model, the equations, initially proposed by (Romeo-Gomez \& Choi, 2011), were adopted concerning the dispersion / diffusion phenomenon, and compare the results modelled with the experimental data obtained by contaminating the water network in the Netherlands. 


\section{Results and Discussion}

The following results show the application of the two models (standard advective EPANET and diffusive-dispersive-advective model) to the UKE (IT) network and to the real network located in Zandvoort (NL). The results show the potential impact of diffusion / dispersion processes with respect to the water quality of the distribution networks.

The simplified advective model does not need calibration as the quality module is a consequence of the hydraulic model parameters that were considered fixed in the present study. The diffusive dispersive - advective approach requires the calibration of the equations (1) - (4) and specifically of foreward and backward dispersion coefficients Ef and $\mathrm{Eb}$.

Calibration process was based on a simple trial and error procedure aiming at the maximization of Nash - Sutcliffe (N-S) convergence criterion among measured concentrations and simulated ones.

Figure 2 compares the experimental and numerical results (with and without dispersion), obtained by contaminating the Unikore network at node 6 with sodium chloride for a duration of 12 minutes and a mass of 370 grams, leading to a constant concentration of $3700 \mathrm{mg} / 1$. In addition, the effect of the Reynolds number on the diffusive-dispersive phenomenon was evaluated. The results shown refer to pipes 8 (a) and 9 (b) of the UKE network.

The two most relevant parameters (the backward and forward dispersion parameters Eb and Ef) were calibrated at 2.6 and 3.1 respectively. The figure shows two cases in which flows are laminar or in transition between laminar and turbulent flow.

It should be noted that in Figure 2a, in the Transition Flow Regime with $\mathrm{Re}=3600$, the agreement between the experimental and diffusive-dispersive-advective results is good. The advective model is not able to represent the process overestimating peaks and missing the delay of concentration peak in time. The N-S criterion in this simulation was equal to 0.96 for the advective-dispersive-diffusive approach and 0.78 for the simple advective approach.

Figure $2 \mathrm{~b}$ shows an experiment in which the Flow Regime is Laminar and Re is equal to 1500 . The diffusion-dispersion phenomenon is more evident and the differences between the two modelling approaches are large both in terms of peak estimation and peak delay. The N-S criterion in this simulation was equal to 0.74 for the advective-dispersive-diffusive approach and negative for the simple advective one demonstrating that this last method is unsuitable to analyse low velocity flows in pipes.

For $\operatorname{Re}>20.000$, the advective approach overestimate the peak concentration of less than $10 \%$; differences between the two models and the experiments become irrelevant for Re values higher than 35.000 .

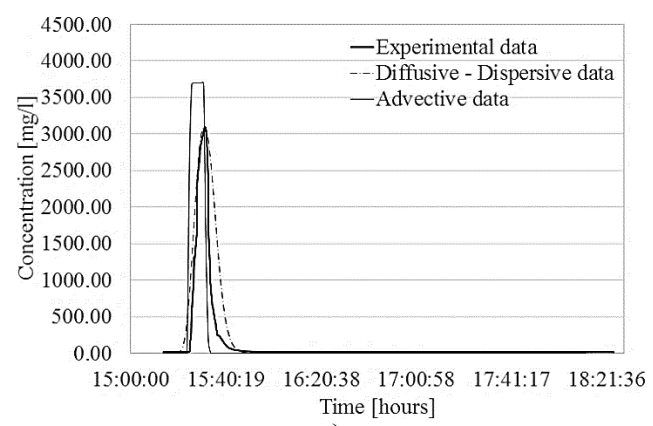

a)

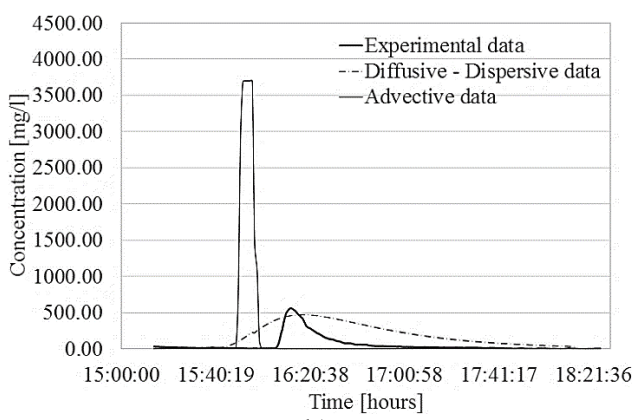

b)

Figure 2: Comparison of experimental, advective and dispersive data for pipes 8 (a) and node 9 (b), having respectively Reynolds number equal to 3598 (Transition Flow Regime), 1542 (Laminar Flow Regime) 
The laboratory experimental investigation allowed to identify the values of Reynolds number for which dispersion and diffusion play a relevant role in contaminant propagation. The experimental campaign also demonstrated that advective simplification is acceptable when turbulent flow is well established. After the laboratory application, the two modelling approaches were applied to the real case study provided by a real water distribution network in Netherlands.

Figure 3 shows the numerical results regarding water age, obtained by comparing the advective solutions determined in the study performed by (Blokker, et al., 2010) and the advective-dispersivediffusive approach presented in this paper for the monitoring station located at Burg. Fennemaplein.

The comparison of the two approaches reports a substantial agreement in terms of water age. This is probably due to the fact that water age computation algorithm is based in both cases on a complete mixing approach at the nodes and dispersion play a modest role only in the evaluation of transit time in the pipes.

If the objective of the study is the evaluation of water detention in the system, the use of complete advective-dispersive-diffusive approach is not justified as the difference is limited to $-12 \%$ in the average with peaks around $-20 \%$. Differently, if the analysis aims to the investigation of contaminant concentrations, the two approaches agree in term of peak concentration and diverge significantly in terms of persistence of water contamination.

Figure 4 shows the results of the simulation and the Electro - Conductivity (EC) measurements during a contamination experiment on 3rd - 4th September 2008, the NaCl-pulse at the inlet location into the network was from 3:40 - 6:40 of the first day. The pulse reached the different locations depending on transfer time between the morning of 3rd of September and the morning of the next day. Particularly, this pulse arrived at 4 September at location Sterflat Friedhoffplein, after 9:00 in the morning. For this reason the figure shows two peaks (the second due to the next contamination experiment) in all locations apart from Sterflat Fridhoffplein. The figures clearly show that the advective approach tends to provide a much shorter contamination with much smaller mass of the contaminant reaching the user. The diffusive approach provides a more realistic distribution of contaminant concentrations respecting peaks and providing a better estimation of contaminant masses. Once again, calibration provided better results for the advective-dispersive-diffusive approach providing higher values of the N-S criterion (respectively 0.79 and 0.88 in the two time-frames presented in figure $4 \mathrm{a}$ and $4 \mathrm{~b}$ ) obtained for a backward dispersion coefficient equal to 2.42 and a forward dispersion coefficient equal to 2.60. Using the same parameters value in the other two locations, $\mathrm{N}-\mathrm{S}$ criterion was equal to 0.68 and 0.71 in the two other locations represented in figure $4 \mathrm{c}$ ) and d).

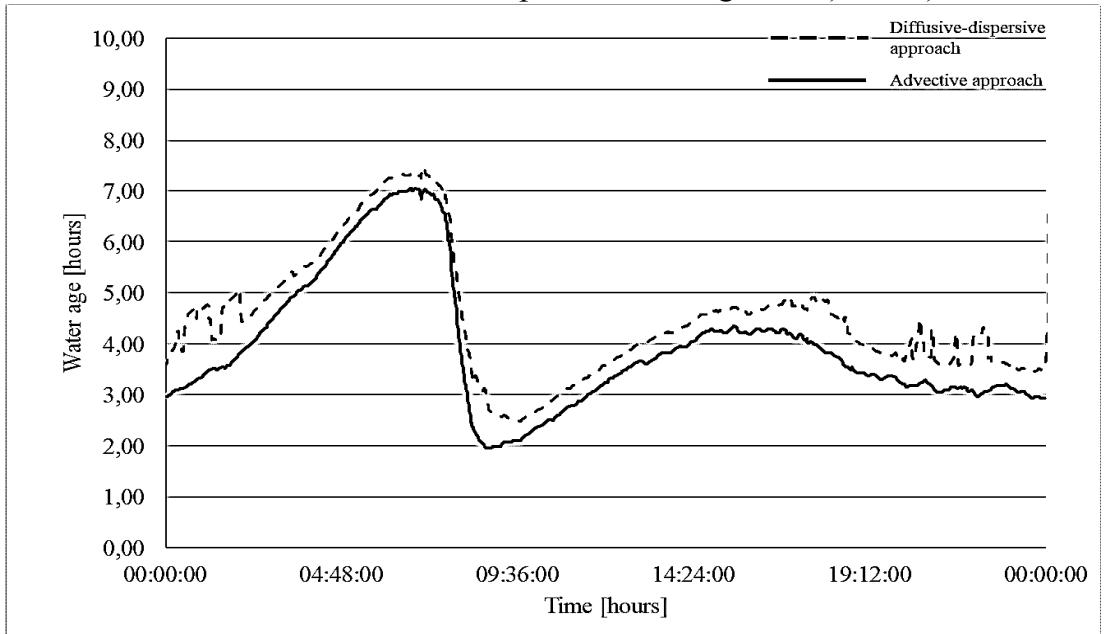

Figure 3: Modelled water age at location Burg. Fennemaplein during 24h 
Lastly, the Reynolds number for the entire network in Zandvoort was determined. It is observed that within 24 hours the speeds are variable depending on the diameters present in the network and the flow rates taken by the users. This makes it oscillate the global network flow regime in a range that assumes values of Reynolds number between a minimum equal to 198 and a maximum equal to 99684 (respectively laminar and fully turbulent).

\section{Conclusion}

The analysis showed how the dispersive and diffusive processes are relevant in the simulation of solute propagation in water networks. The importance of such processes decreases with the presence of turbulence in pipes and they can be considered negligible once Re number reaches values higher than 35.000 and turbulence is stable and established.

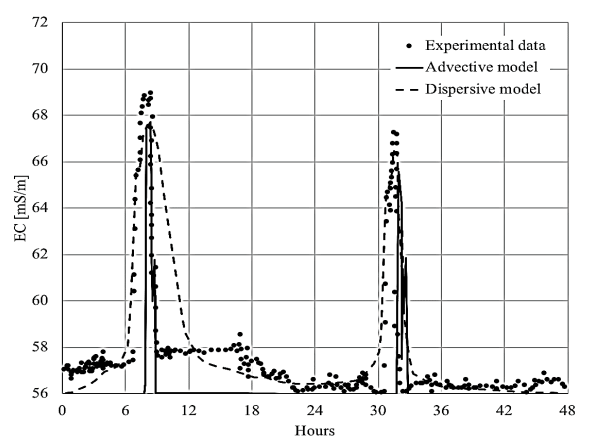

(a)

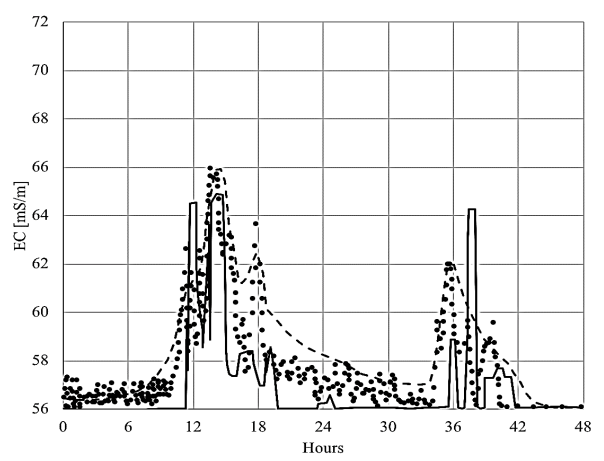

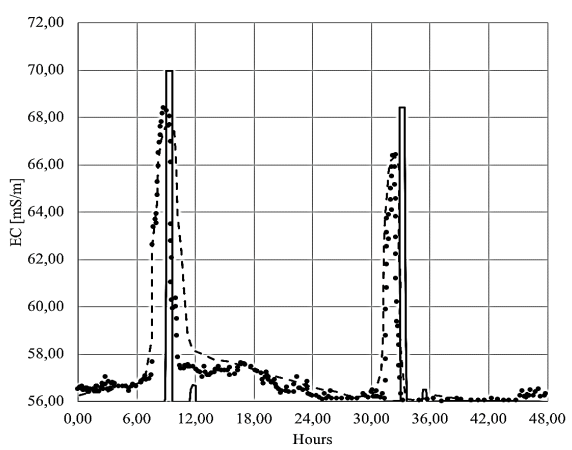

(b)

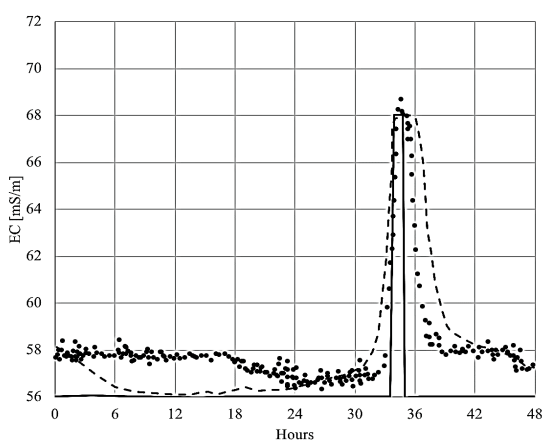

(d)

Figure 4: Comparison EC measurements (Blokker et al., 2010) and simulated with and without dispersion (backward dispersion $=2.42$ and forward dispersion $=2.60)$ for $3^{\text {rd }}-4^{\text {th }}$ September 2008 contamination event at Burg. Fennemaplein (a), De Ruyterstraat (b), NH hotel (c), Sterflat Friefhoffplein (d)

The laboratory analysis was able to highlight the impact of dispersion and diffusion in controlled conditions but the tests on a real network added some interesting additional elements supporting the use of more complex modelling approaches: 
- In real networks, Reynolds numbers may vary significantly during the day and diffusiondispersion may results to have variable impact on contamination;

- Even if peaks are better represented by the complete approach, such model is still uncapable to represent the complexity of the recession limb of the pollutograph;

- The need for calibration of the diffusion-dispersion coefficients requires the availability of real data for calibration even if parameter values are not characterised by significant variations in the two analysed case studies.

\section{References}

Axworthy, D. H. \& Karney, B., 1996. Modelling Low Velocity/High Dispersion Flow in Water Distribution Systems. Journal of Water Resources Planning and Management, Volume 122, pp. 218221.

Berger, J., Gasparin, S., Dutykh, D. \& Mendes, N., 2017. Accurate numerical simulation of moisture front in porous material. Building and Environment.

Blokker, E. J. M. et al., 2010. A bottom-up approach of stochastic demand allocation in water quality modelling. Drinking Water Engineering and Science, Volume 3, pp. 43-51.

Boccelli, D. L. et al., 1998. Optimal Scheduling of Booster Disinfection in Water Distribution Systems. Journal of Water Resources Planning and Management, Volume 124, pp. 99-111.

De Marchis, M. et al., 2016. Experimental Evidence of Leaks in Elastic Pipes. Water Resources Management, Volume 30 (6), pp. 2005-2019.

Dong, W. \& Selvadurai, A. P. S., 2008. A Taylor-Galerkin approach for modelling a spherically symmetric advective-dispersive transport problem. Communications in Numerical Methods in Engineering, Volume 24, pp. 49-63.

Freni, G. \& Sambito, M., 2017. Probabilistic approach to the positioning of water quality sensors in urban drainage networks. International Conference of Urban Drainage.

Gibbs, M. S. et al., 2006. Investigation into the relationship between chlorine decay and water distribution parameters using data driven methods. Mathematical and Computer Modelling, Volume 44, pp. 485-498.

Li, Y. C. \& Cleall, P. J., 2011. Analytical solutions for advective-dispersive solute transport in double-layered finite porous media. International Journal for Numerical and Analytical Methods in Geomechanics, Volume 35, p. 438-460.

Ozdemir, O. N. \& Ucaner, M. E., 2005. Success of booster chlorination for water supply networks with genetic algorithms. Journal of Hydraulic Research, 43(3), pp. 267-275.

Pérez Guerrero, J. S., Pontedeiro, E. M., van Genuchten, M. T. \& Skaggs, T. H., 2013. Analytical solutions of the one-dimensional advection-dispersion solute transport equation subject to timedependent boundary conditions. Chemical Engineering Journal, Volume 221, pp. 487-491.

Piazza, S. et al., 2017. Optimal positioning of sensors in water distribution networks: comparison of numerical and experimental results. Computing and Control for the Water Industry.

Preis, A. \& Ostfeld, A., 2008. Multiobjective Contaminant Sensor Network Design for Water Distribution Systems. Journal of Water Resources Planning and Management, Volume 134, pp. 366377.

Romeo-Gomez, P. \& Choi, C. Y., 2011. Axial Dispersion Coefficients in Laminar Flows of WaterDistribution Systems. Journal of Hydraulic Engineering, pp. 1500-1508.

Tolson, B. A., Maier, H. R., Simpson, A. R. \& Lence, B. J., 2004. Genetic Algorithms for Reliability-Based Optimization of Water Distribution Systems. Journal of Water Resources Planning and Management, Volume 130, pp. 63-72. 
Williams, G. P. \& Tomasko, D., 2008. Analytical Solution to the Advective-Dispersive Equation with a Decaying Source and Contaminant. Journal of Hydrologic Engineering, Volume 13, pp. 1193 1196. 